\title{
Performance of the muon identification and reconstruction with the ATLAS detector
}

\author{
Stefanos Leontsinis ${ }^{1,2, a}$, on behalf of the ATLAS Collaboration \\ ${ }^{1}$ National Technical University of Athens \\ ${ }^{2}$ Brookhaven National Laboratory
}

\begin{abstract}
We present the muon reconstruction algorithms used in ATLAS during the LHC run-1 and their performances in terms of efficiency, muon momentum scale and resolution. These performances have been measured using large calibration samples of $J / \psi, \Upsilon$ and $Z$ decays, which allow to control the systematic uncertainties on efficiency and on momentum scale at the per-mille level. Corrections to be applied to simulation have been derived from the performances measurements and used in physics analyses. The impact of these correction on physics measurements, and the associated uncertainties, is also presented.
\end{abstract}

\section{Introduction}

ATLAS detector [1] is a multipurpose detector ${ }^{1}$ lying in the Large Hadron Collider (LHC) ring [2]. The ATLAS experiment consists of the inner detector (ID), the colorimeter and the muon spectrometer (MS). All three systems are used for the reconstruction of muons.

The ID is the closest system to the interaction point and provide tracking information for all charged particles within $|\eta|<2.5$. ID is inside an axial magnetic field of $2 \mathrm{~T}$ and it consists of the Silicon Pixels, the Semi-Conductor Tracker (SCT) and the Transition Radition Tracker (TRT) detectors.

The calorimeter is located between the ID and the MS. It includes the liquid argon (LAr) electromagnetic calorimeter and the hadronic calorimeter (based on iron and scintillator tiles) providing a coverage of $|\eta|<3.2$ and $|\eta| \leq 1.5$ respectively.

The MS is the outermost of all ATLAS sub-detector systems. It consists of two parts, the barrel $(|\eta|<1.05)$ and the endcaps, covering a range of $|\eta|<2.7$. Both barrel and endcap systems are surrounded by superconducting air-core toroid magnets, providing a magnetic field with a bending integral of about $2.5 \mathrm{Tm}$ and $6 \mathrm{Tm}$ respectively. Two different technologies provide trigger information (Resistive Plate Chambers - RPC in the barrel and Thin Gap Chambers - TGC in the endcaps). Two

\footnotetext{
ae-mail: stefanos.leontsinis@cern.ch

${ }^{1}$ ATLAS uses a right-handed coordinate system with its origin at the nominal interaction point (IP) in the centre of the detector and the $z$-axis along the beam pipe. The $x$-axis points from the IP to the centre of the LHC ring, and the $y$-axis points upward. Cylindrical coordinates $(r, \phi)$ are used in the transverse plane, $\phi$ being the azimuthal angle around the beam pipe. The pseudorapidity is defined in terms of the polar angle $\theta$ as $\eta=-\ln \tan (\theta / 2)$. The distance $\Delta R$ in $\eta-\phi$ space is defined as $\Delta R=\sqrt{\Delta \eta^{2}+\Delta \phi^{2}}$
} 
more types of detectors (Monitored Drift Tubes - MDT and Cathode Strip Chambers - CSC) provide precise muon momentum measurement.

The performance of the muon identification and reconstruction is crucial to the physics program of ATLAS; from standard model precision measurements [3] to the Higgs boson discovery [4] and searches for exotic physics [5].

In these proceedings we present the performance of the ATLAS muon reconstruction using $p p$ collision data at $\sqrt{s}=8 \mathrm{TeV}$ recorded during 2012 [6]. This study (see Ref. [6]) follows an earlier ATLAS publication, using 2010 data [7], where due to the limited statistics, uncertainties were larger.

\section{Muon identification and reconstruction algorithms}

Four types of muon reconstruction criteria are used, based on the available information from the ATLAS sub-detectors (illustrated on Fig. 1). The type of muons that their trajectory is reconstructed using information coming only from MS is called "stand-alone" (SA - see Fig. 1a). The muon track parameters are defined by extrapolating the track to the interaction point, taking into account the energy loss from the calorimeters. This type of muons is used mainly to extend the coverage of the ID $(2.5<|\eta|<2.7)$.

The main type of reconstructed muons in ATLAS are the "combined" muons (CB - see Fig. 1b). The CB muon tracks are formed from a successful combination of a MS with an ID track.

ID tracks that are associated with at least one segment in the MDT or CSC chambers are classified as segment-tagged muons (ST). ST muons (see Fig. 1c) are used in regions with reduced MS acceptance and when, mainly low $p_{\mathrm{T}}$, muons cross only one layer of the MS.

The last type of muons are the "calorimeter-tagged" (CaloTag) muons (see Fig. 1d). These are formed by ID tracks that are associated to an energy deposit in the calorimeter compatible with a minimum ionizing particle. Although this type has the lowest purity, compared to the other three mentioned above, it recovers acceptance in the uninstrumented regions of the MS.

\section{Efficiency measurement}

The efficiency is determined using the "tag and probe" (TP) method in $Z \rightarrow \mu \mu$ and $J / \psi \rightarrow \mu \mu$ decays. For the $Z \rightarrow \mu \mu$, the tag muon, is required to have triggered the event and to be $\mathrm{CB}$. The other muon (probe) is required to be a MS track (SA or CB). After selecting all TP pairs, an attempt is made to match the probe to a reconstructed muon: a match is successful when the muon and the probe are close in the $\eta-\phi$ plane. The efficiency for finding a muon of type $A$ given a probe of type $B$ is given by

$$
\varepsilon(A \mid B)=\frac{N_{\text {Probes }}^{\text {Match }}(\text { Data })-N_{\text {Probes }}^{\text {Match }}(\mathrm{Bkg})}{N_{\text {Probes }}^{\text {All }}(\text { Data })-N_{\text {Probes }}^{\text {All }}(\text { Bkg })},
$$

where $N_{\text {Probes }}^{\text {All }}($ Sample $)$ is the total number of probes considered and $N_{\text {Probes }}^{\mathrm{Match}}($ Sample $)$ is the number of probes successfully matched to a reconstructed muon of type A. "Sample", can be either "Data" or "Bkg", where background is estimated using MC.

The $J / \psi \rightarrow \mu \mu$ decay is used to complement the efficiencies extracted from the $Z \rightarrow \mu \mu$ decays in the low- $p_{\mathrm{T}}$ region. Following the $Z$ TP method, the tag muon from the $J / \psi$ decay must have triggered the event and the probe muon is an opposite charged muon that together with the tag muon form an invariant mass that fall in the window $2.7-3.5 \mathrm{GeV}$. The MS reconstruction efficiency is defined as

$$
\varepsilon(\text { TYPE } \mid \text { ID })=1-\frac{N_{b}}{N_{a}}
$$




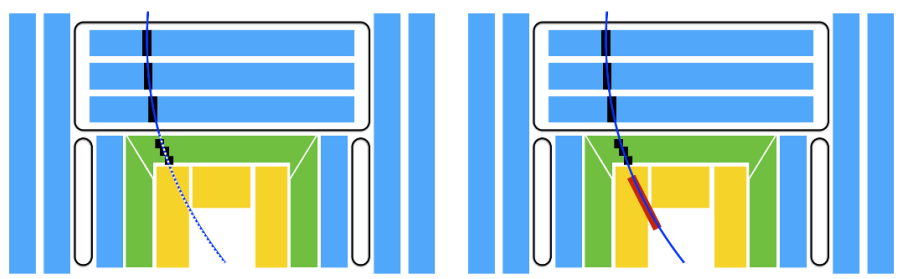

(a)

(b)

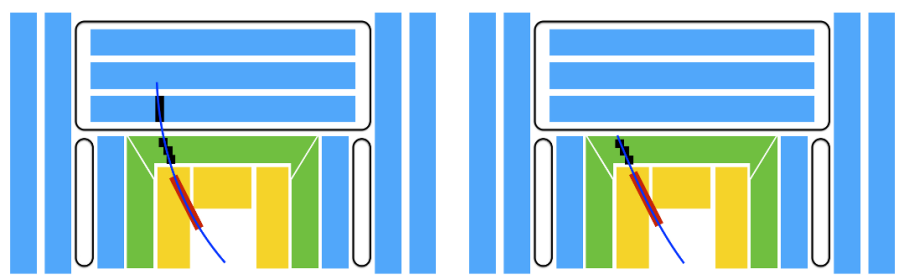

(c)

(d)

Figure 1: Schematic of the different types of muons. (a) Stand-Alone (b) Combined (c) Segmenttagged and (d) Calorimeter-tagged.

where $N_{a}$ and $N_{b}$ are the fitted signal yields of the invariant mass distributions for all TP pairs and TP pairs in which the probe failed to be reconstructed in the MS respectively.

Figure 2 shows the muon reconstruction efficiency as a function of pseudorapidity using $Z \rightarrow$ $\mu \mu$ events for the different reconstruction algorithms. The efficiency in the central $(|\eta|<0.1)$ and transition region $(1.1<\eta<1.3)$ is recovered by using the ST and CaloTag muons. Overall, a uniform muon reconstruction efficiency of about $99 \%$ over all the detector coverage is observed.

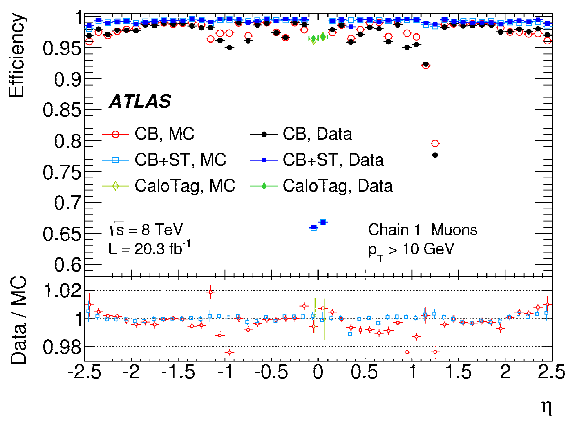

Figure 2: Muon reconstruction efficiency as a function of $\eta$ measured in $Z \rightarrow \mu \mu$ events for different muon reconstruction types. The panel at the bottom shows the ratio between the measured and predicted efficiencies. 
The ID muon reconstruction efficiency as a function of $p_{\mathrm{T}}$ is shown in Fig. 3a. The efficiency is greater that $99 \%$ in the whole $p_{\mathrm{T}}$ range and modelled accurately by the MC. The efficiency for $\mathrm{CB}+\mathrm{ST}$ muons as a function of $p_{\mathrm{T}}$ is shown in Fig. 3b. Results using $J / \psi \rightarrow \mu \mu$ events are shown in the low- $p_{\mathrm{T}}$ region (blue points), followed by the measurements with the $Z \rightarrow \mu \mu$ decays (black points). The drop in efficiency observed using the $J / \psi$ for $p_{\mathrm{T}}>15 \mathrm{GeV}$ is due to the inefficiency of the MS to reconstruct muon pairs with small angular separation coming from highly boosted $J / \psi$ decays.

The reconstruction efficiency is also examined as a function of the mean number of interaction per bunch crossing - $\langle\mu\rangle$ (see Fig. 3c). The efficiency is on average above $99 \%$, very stable and only a very small efficiency drop of about $1 \%$ is observed for the value of $\langle\mu\rangle \geq 35$. This result is very important for the preparation of the detector and the reconstruction algorithms in view of the next LHC run with harsher pileup conditions.

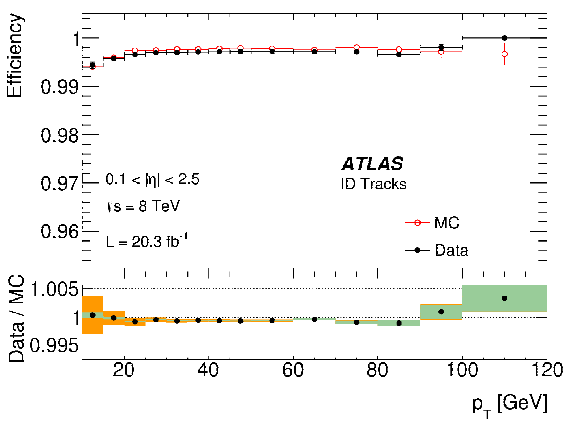

(a)

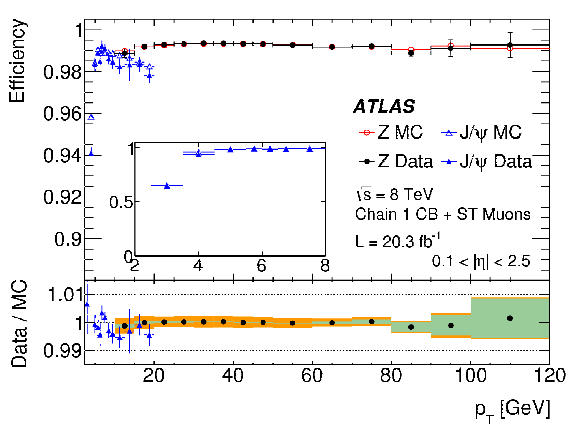

(b)

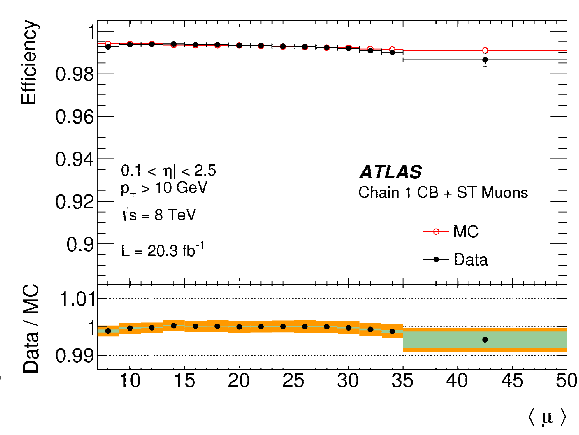

(c)

Figure 3: (a) ID muon reconstruction efficiency as a function of $p_{\mathrm{T}}$ measured in $Z \rightarrow \mu \mu$ events. (b) Reconstruction efficiency for $\mathrm{CB}+\mathrm{ST}$ muons as a function of the muon's $p_{\mathrm{T}}$ using $Z \rightarrow \mu \mu$ and $J / \psi \rightarrow \mu \mu$ events. The insert shows the detail of the efficiency as a function of $p_{\mathrm{T}}$ in the low $p_{\mathrm{T}}$ region. (c) Measured $\mathrm{CB}+\mathrm{ST}$ muon reconstruction efficiency as a function of the average number of inelastic $p p$ collisions per bunch crossing. For all three figures, the panel at the bottom shows the ratio between the measured and predicted efficiencies. 


\section{Mass and momentum resolution}

The big sample of $J / \psi \rightarrow \mu \mu, \Upsilon \rightarrow \mu \mu$ and $Z \rightarrow \mu \mu$ decays allows the study of the muon momentum and the dimuon invariant mass resolution. The invariant mass resolution $\sigma\left(m_{\mu \mu}\right)$ is related to the momentum resolution by

$$
\frac{\sigma\left(m_{\mu \mu}\right)}{m_{\mu \mu}}=\frac{1}{2} \frac{\sigma\left(p_{1}\right)}{p_{1}} \oplus \frac{1}{2} \frac{\sigma\left(p_{2}\right)}{p_{2}}=\frac{1}{\sqrt{2}} \frac{\sigma(p)}{p},
$$

with $p_{1}$ and $p_{2}$ the momenta of the two muons.

The mass resolution is obtained by fitting the width of the invariant mass peaks. The natural widths of the $J / \psi$ and $\Upsilon$ resonances are negligible compared to the experimental resolutions. For the $Z$, the fits performed using a convolution of the true shape, obtained from $\mathrm{MC}$, with an experimental resolution function.

Figure 4 show a comparison of the mass resolution from the $J / \psi, \Upsilon$ and $Z$ dimuon decays in three regions of $|\eta|$. In the barrel region the resolution increases from $\sigma\left(m_{\mu \mu}\right) / m_{\mu \mu}=1.2 \%$ for low- $p_{\mathrm{T}}$ muons to $\sigma\left(m_{\mu \mu}\right) / m_{\mu \mu} \approx 2 \%$ for $p_{\mathrm{T}}=100 \mathrm{GeV}$. For the $|\eta|>1 \sigma\left(m_{\mu \mu}\right) / m_{\mu \mu}$ goes from $2 \%$ to $3 \%$. The results are also reproduced accurately with MC.

Using Eq. 1 we can estimate the relative momentum resolution $\sigma(p) / p$, which ranges from $\approx$ $1.7 \%$ in the central region and low $p_{\mathrm{T}}$ up to $\approx 4 \%$ at forward pseudorapidity and $p_{\mathrm{T}}=100100$.

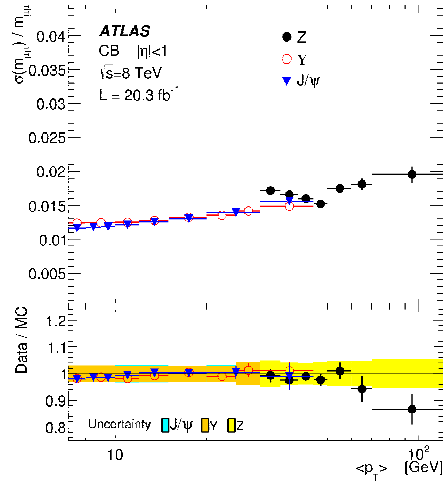

(a)

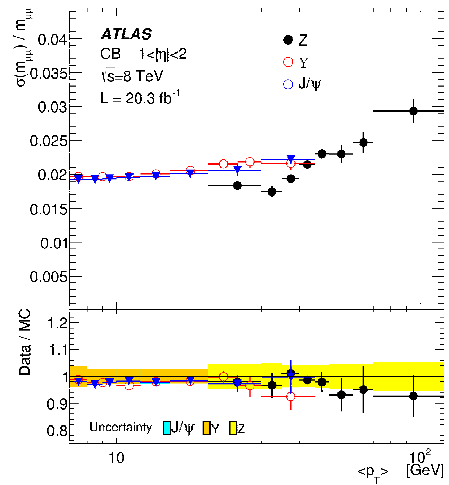

(b)

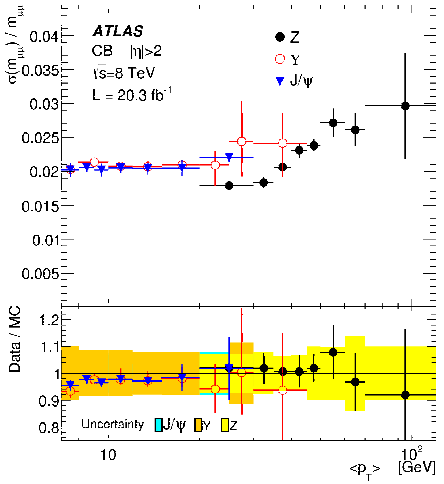

(c)

Figure 4: Dimuon invariant mass resolution for CB muons measured from $J / \psi, \Upsilon$ and $Z$ events as a function of the average transverse momentum in three $|\eta|$ ranges. Both muons are required to be in the same $|\eta|$ range. The lower panel shows the ratio between data and the corrected MC.

\section{Final state radiation recovery}

Final state radiation of photons can cause the mass reconstruction of heavy particles decaying to muons to be shifted to lower values. ATLAS use an FSR recovery method, of which results can be seen in Fig. 5.

FSR photons emitted collinearly to muons are reconstructed with the LAr calorimeter by searching electromagnetic clusters within a narrow cone around the muon momentum direction. In addition, 
non-collinear FSR photons are recovered using the ATLAS photon reconstruction, selecting isolated photons in a cone further away from the muon trajectory. The method is applied in $Z \rightarrow \mu \mu$ events and the effect of recovering collinear and non-collinear photons is shown in Fig. 5a and Fig. 5b respectively. The efficiency of the FSR method is about $70 \%$ for recovering collinear photons and $60 \%$ for non-collinear.

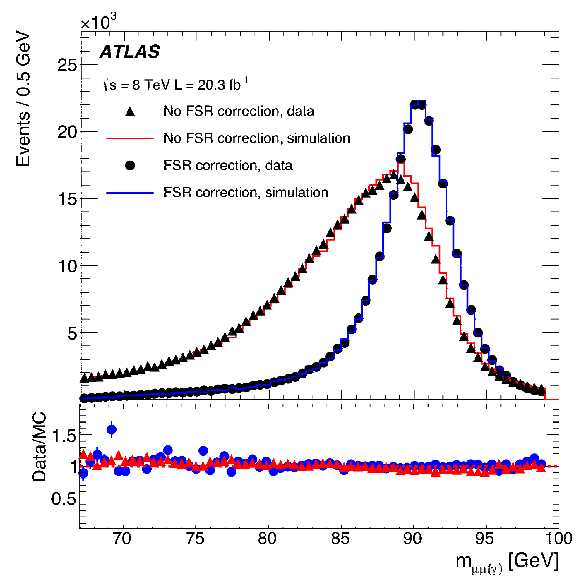

(a)

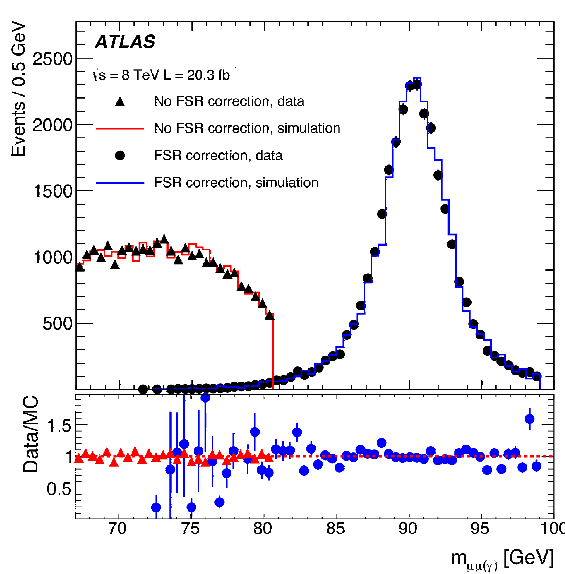

(b)

Figure 5: Invariant mass distribution of $Z \rightarrow \mu \mu$ events with identified FSR in data before (filled triangles) and after (filled circles) FSR correction, for collinear (a) and non-collinear (b) FSR. The $\mathrm{MC}$ prediction is shown before correction (red histogram) and after correction (blue histogram).

\section{Conclusions}

ATLAS muon reconstruction performance has been studied using data from LHC $p p$ collisions at $\sqrt{s}=8 \mathrm{TeV}$ and is $99 \%$ in a pseudorapidity range of $|\eta|<2.5$ and for $p_{\mathrm{T}}>10 \mathrm{GeV}$. Dimuon invariant mass resolution $\sigma\left(m_{\mu \mu}\right) / m_{\mu \mu}$ range from approximately $1 \%$ in the barrel region to $3 \%$ in the endcaps. Finally the FSR recovery allows the recovery of $\approx 4 \%$ of the events.

\section{Acknowledgements}

The present work was co-funded by the European Union (European Social Fund ESF) and Greek national funds through the Operational Program "Education and Lifelong Learning" of the National Strategic Reference Framework (NSRF) 2007-1013. ARISTEIA-1893-ATLAS MICROMEGAS.

\section{References}

[1] ATLAS Collaboration, JINST 3, S08003 (2008).

[2] L. Evans and P. Bryant, JINST 3, S08001 (2008).

[3] ATLAS Collaboration, Phys.Rev. D85, 072004 (2012), arXiv:1109.5141 [hep-ex] . 
[4] ATLAS Collaboration, Phys.Lett. B716, 1 (2012), arXiv:1207.7214 [hep-ex] .

[5] ATLAS Collaboration, Phys.Rev. D87, 015010 (2013), arXiv:1211.1150 [hep-ex] .

[6] ATLAS Collaboration, (2014), arXiv:1407.3935 [hep-ex] .

[7] ATLAS Collaboration, (2014), arXiv:1404.4562 [hep-ex] . 